\title{
Comprehensive profiling of the gut microbiota in patients with chronic obstructive pulmonary disease of varying severity
}

Yu-Chi Chiu

Taoyuan General Hospital

Shih-Wei Lee

Taoyuan General Hospital

Chi-Wei Liu

Taoyuan General Hospital

Rebecca Chou-Jui Lin

Taoyuan General Hospital

Yung-Chia Huang

Taoyuan General Hospital

Tzuo-Yun Lan

National Yang-Ming University

Lawrence Shih-Hsin Wu ( $\triangle$ lshwu@hotmail.com )

China Medical University https://orcid.org/0000-0001-6275-8368

Research article

Keywords: Chronic obstructive pulmonary disease (COPD), severity, gut microbiota, eosinophil

Posted Date: March 23rd, 2020

DOI: https://doi.org/10.21203/rs.3.rs-18063/v1

License: (c) (i) This work is licensed under a Creative Commons Attribution 4.0 International License.

Read Full License 


\section{Abstract}

Background Chronic obstructive pulmonary disease (COPD) is a chronic respiratory disease that reduces lung and respiratory function, with a high mortality rate. Severe and acute deterioration of COPD can easily lead to respiratory failure, resulting in personal, social, and medical burden. Recent studies have shown a high correlation between the gut microbiota and lung inflammation. In this study, we investigated the relationship between gut microbiota and COPD severity.

Results A total of 60 COPD patients with varying severity according to GOLD guidelines were enrolled in this study. DNA was extracted from patients' stool and 16S rRNA data analysis conducted using highthroughput sequencing followed by bioinformatics analysis. The richness of the gut microbiota was not associated with COPD severity. The gut microbiome is more similar in stage 1 and 2 COPD than stage 3+4 COPD. Fusobacterium and Aerococcus were more abundant in stage 3+4 COPD. Ruminococcaceae NK4A214 group and Lachnoclostridium were less abundant in stage 2-4, and Tyzzerella 4 and Dialister were less abundant in stage 1. However, the abundance of a Bacteroides strain was associated with eosinophil count and lung function.

Conclusions This study suggests that no distinctive gut microbiota pattern is associated with the severity of COPD. The gut microbiome could affect COPD by gut inflammation shaping the host immune system.

\section{Background}

Chronic obstructive pulmonary disease (COPD) is an inflammatory lung disorder characterized by the progressive obstruction of airflow, which leads to symptoms such as shortness of breath, cough, and increased sputum production [1]. Exacerbation of COPD often results in high mortality and morbidity, rapid decline in lung function, and increased health care expense [2]. Though cigarette smoking is associated with COPD, not all smokers develop the disease [1]. Furthermore, even though COPD can lead to exacerbations, not all patients are susceptible to the symptoms. Therefore, COPD is a heterogeneous disease that may be affected by multiple factors that are not yet fully understood.

The pathogenesis of COPD is thought to involve inflammatory mediators and bacterial or viral infections [3]. In particular, systemic inflammation [4] and airway inflammation [5] are often associated with exacerbation. Traditional culturing techniques have already found evidence of bacterial and viral colonization in the airways of COPD patients between exacerbations [6, 7]. These pathogens persist in the respiratory tract, creating a diverse environment in the airways and lungs. Though their presence in relation to exacerbations is not yet clearly defined, it has been postulated that any pathogen exposure may enhance surfactant abnormalities, hinder mucociliary clearance, and increase the patient's susceptibility to chronic inflammation, worsening respiratory symptoms and accelerating disease progression.

This gut dysbiosis in humans has been linked to inflammatory conditions in the gastrointestinal tract itself, but also in the airways, such as in asthma and COPD [8, 9]. Accumulating evidence has highlighted 
the influence of the gut microbiota on lung immunity, referred to as the gut-lung axis, though the underlying pathways and mechanisms are still areas of intensive research [10]. Short-chain fatty acids (SCFAs), as a metabolic by-products derived from bacterial fermentation of dietary fibers, are the most extensively studied metabolites, and have variant immunomodulatory functions of host physiology [11].

The gut microbiome-dependent and dietary-associated metabolite trimethylamine-N-oxide (TMAO) has been shown to be a risk-predicting biomarker for atherosclerosis and incident cardiovascular events [1215]. In addition, an independent association of TMAO with long-term all-cause mortality has been found in patients with congestive heart failure (CHF), chronic kidney disease (CKD), community-acquired pneumonia, and COPD [16-19].COPD patients are at higher risk for cardiovascular diseases [20], which are the most likely cause of death in patients with mild to moderate COPD [21].

Several studies have found significant differences in the distribution of respiratory microbiota between healthy individuals and COPD patients, and between different levels of COPD severity [22]. There has been growing interest in addressing the effect of probiotics on lung disorders, such as asthma and COPD [23], which should indicate whether the gut microbiome is associated with COPD exacerbation or severity. In the present study, we investigated the relationship between gut microbiota and COPD severity.

\section{Results}

\section{Study subjects}

We totally enrolled 60 male COPD patients and 20 patients diagnosed as stage 1,20 patients as stage 2 , and 20 patients as stage $3+4$. The demographic and clinical features of enrolled patients were listed in Table 1. The patients in mild COPD group were elder than patients in moderate and severe COPD groups. Clinical features (with statistical significance), such as pulmonary function test, reflected the different among this three patients groups. The medication also had significant difference among the three study groups 
Table 1

The demographic and clinical characteristics of the study participants

\begin{tabular}{|c|c|c|c|c|}
\hline Variables & Mild COPD & Moderate COPD & Severe COPD & $p$ value \\
\hline \multicolumn{5}{|l|}{ Age (years) } \\
\hline Mean \pm SD (range) & $78 \pm 11(51-95)$ & $72 \pm 10(51-91)$ & $68 \pm 8(50-81)$ & $0.008^{a}$ \\
\hline \multicolumn{5}{|l|}{$\mathrm{BH}(\mathrm{m})$} \\
\hline Mean \pm SD & $1.64 \pm 0.07$ & $1.66 \pm 0.07$ & $1.63 \pm 0.07$ & $0.630^{\mathrm{a}}$ \\
\hline \multicolumn{5}{|l|}{ BW (Kg) } \\
\hline Mean \pm SD & $61.79 \pm 9.75$ & $63.86 \pm 10.24$ & $58.94 \pm 8.88$ & $0.277^{a}$ \\
\hline \multicolumn{5}{|l|}{ BMI } \\
\hline Mean \pm SD & $22.85 \pm 3.51$ & $23.35 \pm 3.71$ & $22.09 \pm 3.40$ & $0.535^{\mathrm{a}}$ \\
\hline \multicolumn{5}{|l|}{ WBC (per ul) } \\
\hline Median (range) & $6700(4090-9060)$ & $\begin{array}{l}6470(4620- \\
13740)\end{array}$ & $\begin{array}{l}9335(2580- \\
19590)\end{array}$ & $0.081^{b}$ \\
\hline \multicolumn{5}{|l|}{ Eosinophil (\%) } \\
\hline Median (range) & $2.10(0.5-10.9)$ & $1.75(0-8.4)$ & $1.60(0-14.8)$ & $0.354^{\mathrm{b}}$ \\
\hline \multicolumn{5}{|l|}{ Eosinophil (per ul) } \\
\hline Median (range) & $146(29-701)$ & $118(0-597)$ & $162(0-881)$ & $0.216^{b}$ \\
\hline \multicolumn{5}{|l|}{$\operatorname{lgE}(k U / L)$} \\
\hline Median (range) & $\begin{array}{l}53.45(1.50- \\
7758.30)\end{array}$ & $20.80(1.5-436.30)$ & $\begin{array}{l}62.50(6.80- \\
2130.30)\end{array}$ & $0.296^{b}$ \\
\hline \multicolumn{5}{|l|}{ Smoking (n) } \\
\hline Yes & 15 & 17 & 18 & $0.432^{\mathrm{c}}$ \\
\hline No & 5 & 3 & 2 & \\
\hline
\end{tabular}

$\mathrm{SD}=$ standard deviation; $\mathrm{COPD}=$ chronic obstructive pulmonary disease; $\mathrm{n}=$ number of subjects; $\mathrm{BH}=$ body height; $\mathrm{WB}=$ body weight; $\mathrm{BMI}=$ body mass index; $\mathrm{WBC}=$ white blood cell; $\mathrm{CAT}=\mathrm{COPD}$ Assessment Test; $\mathrm{mMRC}=$ Modified Medical Research Council; FVC = forced vital capacity; FEV1 = Forced expiratory volume in one second; LAMA = long-acting muscarinic antagonist; LABA = longacting beta agonist; ICS = inhaled corticosteroid; a: The statistical analysis was tested by One-way ANOVA; b: The statistical analysis was tested by Kruskal-Wallis test; c: The statistical analysis was tested by $\chi 2$-test.

Figure 1 


\begin{tabular}{|c|c|c|c|c|}
\hline Variables & Mild COPD & Moderate COPD & Severe COPD & $p$ value \\
\hline \multicolumn{5}{|l|}{ CAT } \\
\hline Mean \pm SD & $6.60 \pm 3.50$ & $9.45 \pm 6.92$ & $14.25 \pm 6.30$ & $\begin{array}{l}< \\
0.001^{\mathrm{a}}\end{array}$ \\
\hline Score < 10 (n) & 17 & 12 & 6 & $0.002^{c}$ \\
\hline Score $\geqq 10(n)$ & 3 & 8 & 14 & \\
\hline \multicolumn{5}{|l|}{ mMRC } \\
\hline Mean \pm SD & $0.35 \pm 0.59$ & $1.05 \pm 1.19$ & $1.80 \pm 1.06$ & $\begin{array}{l}< \\
0.001^{\mathrm{a}}\end{array}$ \\
\hline Score < 2 (n) & 19 & 12 & 8 & $0.001^{c}$ \\
\hline Score $\geqq 2(n)$ & 1 & 8 & 12 & \\
\hline \multicolumn{5}{|c|}{$\begin{array}{l}\text { Pulmonary function } \\
\text { test }\end{array}$} \\
\hline \multicolumn{5}{|c|}{ Pre-bronchodilator } \\
\hline \multicolumn{5}{|l|}{ FVC (L) } \\
\hline Mean \pm SD & $3.18 \pm 0.61$ & $2.88 \pm 0.65$ & $2.48 \pm 0.54$ & $0.002^{a}$ \\
\hline \multicolumn{5}{|c|}{ FVC (\% predicted) } \\
\hline Mean \pm SD & $115.65 \pm 24.85$ & $96.45 \pm 17.70$ & $84.80 \pm 21.21$ & $\begin{array}{l}< \\
0.001^{\mathrm{a}}\end{array}$ \\
\hline \multicolumn{5}{|l|}{$\mathrm{FEV}_{1}(\mathrm{~L})$} \\
\hline Mean \pm SD & $2.05 \pm 0.48$ & $1.52 \pm 0.39$ & $0.97 \pm 0.24$ & $\begin{array}{l}< \\
0.001^{\mathrm{a}}\end{array}$ \\
\hline \multicolumn{5}{|c|}{$\mathrm{FEV}_{1}(\%$ predicted $)$} \\
\hline Mean \pm SD & $98.40 \pm 20.23$ & $65.95 \pm 13.30$ & $42.15 \pm 11.38$ & $\begin{array}{l}< \\
0.001^{\mathrm{a}}\end{array}$ \\
\hline
\end{tabular}

$\mathrm{SD}=$ standard deviation; $\mathrm{COPD}=$ chronic obstructive pulmonary disease; $\mathrm{n}=$ number of subjects; $\mathrm{BH}=$ body height; $\mathrm{WB}=$ body weight; $\mathrm{BMI}=$ body mass index; $\mathrm{WBC}=$ white blood cell; $\mathrm{CAT}=\mathrm{COPD}$ Assessment Test; $\mathrm{mMRC}=$ Modified Medical Research Council; FVC = forced vital capacity; FEV1 = Forced expiratory volume in one second; LAMA = long-acting muscarinic antagonist; LABA = longacting beta agonist; ICS = inhaled corticosteroid; a: The statistical analysis was tested by One-way ANOVA; b: The statistical analysis was tested by Kruskal-Wallis test; c: The statistical analysis was tested by $\chi 2$-test.

Figure 1 


\begin{tabular}{|c|c|c|c|c|}
\hline Variables & Mild COPD & Moderate COPD & Severe COPD & $p$ value \\
\hline \multicolumn{5}{|l|}{$\mathrm{FEV}_{1} / \mathrm{FVC}$ ratio (\%) } \\
\hline Mean \pm SD & $64.80 \pm 11.38$ & $54.05 \pm 13.18$ & $40.10 \pm 10.85$ & $\begin{array}{l}< \\
0.001^{\mathrm{a}}\end{array}$ \\
\hline \multicolumn{5}{|c|}{ Post-bronchodilator } \\
\hline \multicolumn{5}{|l|}{$\mathrm{FVC} \pm \mathrm{SD}(\mathrm{L})$} \\
\hline Mean \pm SD & $3.33 \pm 0.51$ & $3.03 \pm 0.66$ & $2.64 \pm 0.64$ & $0.003^{a}$ \\
\hline \multicolumn{5}{|l|}{ FVC (\% predicted) } \\
\hline Mean \pm SD & $120.75 \pm 19.30$ & $101.85 \pm 18.23$ & $90.05 \pm 22.60$ & $\begin{array}{l}< \\
0.001^{\mathrm{a}}\end{array}$ \\
\hline \multicolumn{5}{|l|}{$\mathrm{FEV}_{1}(\mathrm{~L})$} \\
\hline Mean \pm SD & $2.10 \pm 0.43$ & $1.63 \pm 0.39$ & $1.03 \pm 0.27$ & $\begin{array}{l}< \\
0.001^{\mathrm{a}}\end{array}$ \\
\hline \multicolumn{5}{|l|}{$\mathrm{FEV}_{1}(\%$ predicted $)$} \\
\hline Mean \pm SD & $101.30 \pm 18.41$ & $71.20 \pm 13.59$ & $44.60 \pm 12.48$ & $\begin{array}{l}< \\
0.001^{\mathrm{a}}\end{array}$ \\
\hline \multicolumn{5}{|l|}{$\mathrm{FEV}_{1} / \mathrm{FVC}$ ratio (\%) } \\
\hline Mean \pm SD & $63.15 \pm 7.97$ & $54.80 \pm 11.44$ & $40.10 \pm 11.38$ & $\begin{array}{l}< \\
0.001^{a}\end{array}$ \\
\hline \multicolumn{5}{|l|}{ Medication } \\
\hline LAMA (n) & 10 & 3 & 0 & $0.005^{c}$ \\
\hline $\operatorname{LABA}(\mathrm{n})$ & 1 & 1 & 0 & \\
\hline LAMA + LABA (n) & 4 & 7 & 8 & \\
\hline ICS + LABA (n) & 3 & 3 & 1 & \\
\hline
\end{tabular}

$\mathrm{SD}=$ standard deviation; $\mathrm{COPD}=$ chronic obstructive pulmonary disease; $\mathrm{n}=$ number of subjects; $\mathrm{BH}=$ body height; $\mathrm{WB}=$ body weight; $\mathrm{BMI}=$ body mass index; $\mathrm{WBC}=$ white blood cell; $\mathrm{CAT}=\mathrm{COPD}$ Assessment Test; $\mathrm{mMRC}=$ Modified Medical Research Council; FVC = forced vital capacity; FEV1 = Forced expiratory volume in one second; LAMA = long-acting muscarinic antagonist; LABA = longacting beta agonist; ICS = inhaled corticosteroid; a: The statistical analysis was tested by One-way ANOVA; b: The statistical analysis was tested by Kruskal-Wallis test; c: The statistical analysis was tested by $\chi 2$-test.

Figure 1 


\begin{tabular}{|c|c|c|c|c|}
\hline Variables & Mild COPD & Moderate COPD & Severe COPD & $p$ value \\
\hline $\begin{array}{l}\text { ICS + LAMA + LABA } \\
\text { (n) }\end{array}$ & 2 & 6 & 11 & \\
\hline \multicolumn{5}{|c|}{ 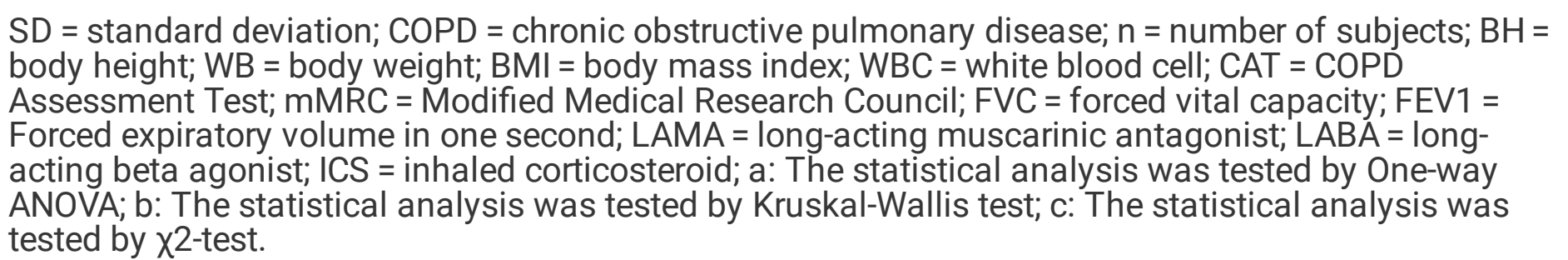 } \\
\hline \multicolumn{5}{|l|}{ Figure 1} \\
\hline
\end{tabular}

\section{Otus}

According to the results of the OTU cluster analysis, the common and unique OTUs of different samples/groups were analysed. The number of samples/groups was 3 , and a Venn diagram of their overlap is shown in Fig. 1. A total of 384 species were found in all three groups. The statistics for the sequence number in each sample OUT are given in Additional file 1: Table S1.

\section{No Significant Difference In Community Richness}

The Chao1 index and Shannon index were used to evaluate the community richness of the microbiome in the three COPD groups. The results are given in Fig. 2. We did not find any significant difference in community richness between groups.

\subsection{Group Similarity at Genus and Species Levels}

The top 30 species in each sample or group were clustered and plotted in a heatmap at the species and genus level (Fig. 3 and Fig. 4). The samples of three groups did not form a distinct cluster according to the cluster of sample analysis in species and genus level (Fig. 3A and Fig. 4A). In the cluster of groups analysis, groups $A$ and $B$ were more similar than group $C$ (Fig. 3B and Fig. 4B). In genus level analysis (Fig. 4B), Fusobacterium and Aerococcus were more abundant in group $\mathrm{C}$ (stage 3 and 4). The Ruminococcaceae NK4A214 group and Lachnoclostridium were less abundant in group B/C (stage 2-4), and Tyzzerella 4 and Dialister were less abundant in group A (stage 1).

\section{Differential Abundance}

The differential analysis was carried out at the strain level. The abundance distribution of the five strains with the largest between-group difference is shown in Fig. 5. The X-axis indicates the names of the five strains and the $\mathrm{Y}$-axis the relative abundance of each. We found four strains, including Veillonella, 
Corynebacterium 1, Romboutsia, and Aerococcus, that are more abundant in group $\mathrm{C}$ than groups $\mathrm{A}$ and B. Megasphaera was found at lower abundance in group A than groups B and C. The statistical significance may due to few outliers.

\section{Otu Abundance Correlated With Eosinophil Count And Lung Function}

Correlation analysis revealed that some OTUs are associated with clinical features (Fig. 6). OTU 19 (Bacteroides sp.) had a stronger negative correlation with eosinophil count $(P<0.001)$ and positively correlated with FEV1 and FVC $(\mathrm{P}<0.05)$. The correlation analysis and $\mathrm{P}$ - values are given in Additional file 2: Table S2.

\section{Discussion}

COPD is becoming a leading cause of death and is increasingly prevalent worldwide $[3,4]$.The full spectrum of factors and mechanisms underlying the disease is still not completely understood. As inflammation induced by bacterial infections is thought to play a role in COPD [17], evidence is emerging that variations in the microbiome may be associated with the progression of COPD. In this study, we investigated the gut microbiome in stool samples from 60 COPD patients with varying severity using $16 \mathrm{~S}$ rRNA gene sequencing. No significant differences in community richness were found between study groups. Fusobacterium and Aerococcus were more abundant in stage $3+4$ COPD. The Ruminococcaceae NK4A214 group and Lachnoclostridium were less abundant in stage $2-4$, and Tyzzerella 4 and Dialister were less abundant in stage 1 . The abundance of a Bacteroides strain was associated with eosinophil count and lung function.

The gut microbiome has not been characterized previously in COPD patients. However, gut bacterial dysbiosis has been reported in response to cigarette smoke in both humans and mice. In human studies, current smokers have increased Bacteroidetes and decreased Firmicutes and Proteobacteria in gut microbiota composition compared to never smokers [24]. Another study revealed that healthy smokers harbour higher Bacteroides-Prevotella than non-smokers [25]. Significant alterations in microbiota composition have been reported in healthy smokers, which reverse upon smoking cessation, with marked increases in both overall microbial diversity and in the phyla Firmicutes and Actinobacteria, and a reduced proportion of Bacteroidetes and Proteobacteria compared to continuing smokers and nonsmokers [26]. Colonic bacterial dysbiosis was reported in mice chronically (24 weeks) exposed to cigarette smoke, with increases in Lachnospiraceae sp. [27]. In our study, Bacteroidetes was more abundant in grade 1 than grade 2-4 COPD (Additional file 3: Figure S1). From the observation of Fig. 5, the present of the abundance distribution of the five strains with the largest between-group difference may due to few outlier(s). That may indicate two issue: (1) the result is suspicious due to random sampling effect; (2) the outlier(s) observed only one or two study groups (not random sampling effect) suppose the bacteria strain(s) associated with COPD severity only in part (not all) patients. However, the 
distinct pattern of gut microbiota defined by one or few bacteria strain is not revealed in this study. Furthermore, the severe COPD patients were higher ratio with inhaled corticosteroid (ICS) treatment that may indicate the medication did not alter gut microbiota obviously.

The blood eosinophil count is associated with the risk of COPD exacerbation, mortality, decreased FEV1, and response to both inhaled and systemic corticosteroids [28]. The differential expression of the airway microbiome between eosinophilic and non-eosinophilic patients with COPD, during both stable disease [29] and acute disease exacerbation [30], suggest that dysregulation of this complex homoeostatic immunity is likely to feature in the pathogenesis of COPD. Bacterial counts for potentially pathogenic microorganisms negatively correlated with sputum eosinophil count, but not blood eosinophil count [31]. Our results indicate that the Bacteroides strain(s) were associated with blood eosinophil count and lung function in COPD. This observation may indicate the different roles of gut and airway microbiomes in COPD via eosinophil inflammation. In previous mouse studies, the gut microbiome was essential to shaping the host immune system [32,33].The gut microbiome should affect COPD by modulating the blood eosinophil count to shape the host immune system, not directly by pathogenic infection.

In our study, Fusobacterium and Aerococcus were more abundant in severe COPD (stage $3+4$ ). Fusobacterium nucleatum is abundant in patients suffering from chronic gut inflammation, contributing to the pathogenesis of colorectal cancer [34]. Aerococcus urinae and Aerococcus sanguinicola are found in human urine and can cause urinary tract infections [35] but are not known in gut pathogenesis. We also found that the Ruminococcaceae NK4A214 group and Lachnoclostridium were less abundant, and Tyzzerella 4 and Dialister more abundant in stage 2-4. In addition, Ruminococcaceae abundance has been demonstrated to be significantly lower in non-alcoholic fatty liver disease (NAFLD) patients than non-NAFLD patients [36], and relatively decreased in bipolar disorder [37]. The relative abundance of Lachnoclostridium, Tyzzerella subgroup 4, Flavonifractor, and unidentified Lachnospiraceae is decreased in autism spectrum disorders [38]. Dialister has been observed at low frequency in non-inflamed ileal and colonic biopsy tissue from patients with spondyloarthritis and healthy controls [39]. Based on the aforementioned studies, we suggest that the bacterial strains in the gut associated with different abundance at varying COPD severity in this study involve gut inflammation.

\section{Conclusion}

Although the gut microbiome has not been characterized in COPD patients previously, the revolution in culture-independent microbiology has yielded tools capable of determining its contribution to the pathogenesis of COPD. Our results found the relationship between gut microbiota and severity of COPD in humans. Furthermore, the association between blood eosinophil count and gut microbiota was also revealed in our study. This should be useful information for developing new diagnostic or therapeutic methods to control COPD progression.

\section{Methods}




\section{Subjects}

A total of 60 COPD patients ( $>20$ years old) with varying severity according to GOLD guidelines [40] were enrolled in this study. DNA was extracted from patients' stool. Patients with cancer or other immunerelated diseases and viral infections (e.g., Hepatitis B, Hepatitis C, HIV, etc.) were excluded from this study. All enrolled subjects lived in urban region (Taoyuan City) and had similar eating habits.

The stool samples were obtained from patients with moderate COPD and patients with severe COPD in stable condition (at least 3 months without exacerbation or use of antibiotics for any other reason). Diagnosis and classification of COPD was established according to GOLD recommendations [40]. The patient groups were defined as A (stage 1), B (stage 2), and C (stage $3+4$ ) according to the classification of airflow limitation in the severity of COPD (2018 GOLD). DNA was extracted from the sputum samples and subjected to next-generation sequencing (NGS). DNA quality was verified before and after rRNA depletion treatment by the Agilent 2100 Bioanalyzer. The DNA samples were also treated with RNase.

The study protocol conformed to the ethical guidelines of the 1975 Declaration of Helsinki and was approved by the Ethics Committee of Taoyuan General Hospital, Taoyuan, Taiwan. Written informed consent was obtained from each patient enrolled in the study.

\section{Metavx ${ }^{\mathrm{m}}$ Library Preparation And Illumina Miseq Sequencing}

NGS library preparations and Illumina MiSeq sequencing were conducted at GENEWIZ, Inc. (Suzhou, China). DNA samples were quantified using a Qubit 2.0 Fluorometer (Invitrogen, Carlsbad, CA, USA). A total of 30-50 ng of DNA was used to generate amplicons using a MetaVx ${ }^{\mathrm{TM}}$ Library Preparation kit (GENEWIZ, Inc., South Plainfield, NJ, USA).

V3 and V4 hypervariable regions of prokaryotic 16S rDNA were selected to generate amplicons and subsequent taxonomy analysis. GENEWIZ designed a panel of proprietary primers aimed at relatively conserved regions bordering the V3 and V4 hypervariable regions of bacteria and Archaea16S rDNA. The $\mathrm{V} 3$ and $\mathrm{V} 4$ regions were amplified using forward primers containing the sequence CCTACGGRRBGCASCAGKVRVGAAT and reverse primers containing the sequence GGACTACNVGGGTWTCTAATCC. First-round PCR products were used as templates for second-round amplicon enrichment PCR. At the same time, indexed adapters were added to the ends of the 16S rDNA amplicons to generate indexed libraries ready for downstream NGS on Illumina Miseq.

The DNA libraries were validated by an Agilent 2100 Bioanalyzer (Agilent Technologies, Palo Alto, CA, USA) and quantified using a Qubit 2.0 Fluorometer. DNA libraries were multiplexed and loaded on an Illumina MiSeq instrument according to the manufacturer's instructions (Illumina, San Diego, CA, USA). Sequencing was performed using a $2 \times 300$ paired-end (PE) configuration; image analysis and base calling were conducted by the MiSeq Control Software (MCS) embedded in the MiSeq instrument. 


\section{Data Analysis}

The QIIME data analysis package was used for 16S rRNA data analysis. The forward and reverse reads were joined and assigned to samples based on barcode, and truncated by cutting off the barcode and primer sequence. Quality filtering of joined sequences was performed and sequences that did not fulfil the following criteria were discarded: sequence length $<200 \mathrm{bp}$, no ambiguous bases, mean quality score $\geq 20$. The sequences were then compared to the reference database (RDP Gold database) using the UCHIME algorithm to detect chimeric sequences, and the chimeric sequences removed. The effective sequences were used in the final analysis. Sequences were grouped into operational taxonomic units (OTUs) using the clustering program VSEARCH (1.9.6) against the Silva 119 database pre-clustered at $97 \%$ sequence identity. The Ribosomal Database Program (RDP) classifier was used to assign a taxonomic category to all OTUs at a confidence threshold of 0.8. The RDP classifier uses the Silva 132 database, which has taxonomic categories predicted to the species level. Sequences were rarefied prior to calculation of alpha and beta diversity statistics. Alpha diversity indexes were calculated in QIIME from rarefied samples using the Shannon index for diversity and the Chao1 index for richness.

Differential analysis of species composition between groups can be performed based on the differential abundance between different groups. Differences in the abundance of microbial communities in two groups can be evaluated using strict statistical methods. The multiple hypothesis test was performed and false discovery rate (FDR) of the rare frequency data determined to evaluate the significance of the observed difference. This analysis was carried out at strain levels.

Correlation analysis uses statistical models to study the correlation between random variables and investigates whether a dependency exits between the phenomena and the nature and level of association. Spearman correlation coefficient was determined using R based on the OTU abundance and environmental factors. P values were also obtained. Heatmaps were generated to illustrate the relationship between clinical features (eosinophil count and lung function) and OTUs.

\section{Declarations}

Competing interests

The authors report no competing interest

Funding

This work was supported by Taoyuan General Hospital, Ministry of Health and Welfare, Taoyuan, Taiwan (PTH10702). The funding bodies had no role in designing the study, sample collection, analysis, and interpretation of data or in writing the manuscript.

Authors' contributions

LSW and TYL performed the analysis and prepared the manuscript. LSW, SWL and YCC designed the project. YCC, SWL, CWL, RCL and YCH collected the study samples and conducted the experiments. LSW supervised the project and revised the manuscript. TYL reviewed the manuscript. All authors read and approved the final version of the manuscript. 
Acknowledgements

Not applicable

Ethics approval and consent to participate

The study protocol was approved by the Ethics Committee of Taoyuan General Hospital, Taoyuan, Taiwan (Approval number: TYGH107039). Written informed consent was obtained from each patient enrolled in the study.

Consent for publication

Not applicable

Availability of data and materials

The datasets used and/or analysed during the current study are available from the corresponding author on reasonable request.

\section{References}

1.

Gold PM. The 2007 GOLD Guidelines: a comprehensive care framework. Respir care. 2009;54:1040-9.

2.

Wedzicha JA, Seemungal TAR. COPD exacerbations: defining their cause and prevention. Lancet. 2007;370:786-96.

3.

Han MK, Huang YJ, Lipuma JJ, Boushey HA, Boucher RC, Cookson WO, Curtis JL, Erb-Downward J, Lynch SV, Sethi S, Toews GB, Young VB, Wolfgang MC, Huffnagle GB, Martinez FJ. Significance of the microbiome in obstructive lung disease. Thorax. 2012;67:456-63.

4.

Perera WR, Hurst JR, Wilkinson TMA, Sapsford RJ, Mullerova H, Donaldson GC, Wedzicha JA. Inflammatory changes, recovery and recurrence at COPD exacerbation. Eur Respir J. 2007;29:527-34.

5 .

Papi A, Bellettato CM, Braccioni F, Romagnoli M, Casolari P, Caramori G, Fabbri LM, Johnston SL. Infections and airway inflammation in chronic obstructive pulmonary disease severe exacerbations. Am $\mathrm{J}$ Resp Crit Care. 2006;173:1114-21.

6.

Hirschmann JV. Do bacteria cause exacerbations of COPD? Chest. 2000;118:193-203.

7.

Wedzicha JA. Role of viruses in exacerbations of chronic obstructive pulmonary disease. Proc Am Thorac Soc. 2004; 1:115-20.

8. 
Rapozo DC, Bernardazzi C, de Souza HS. Diet and microbiota in inflammatory bowel disease: the gut in disharmony. World J Gastroenterol. 2017;23:2124-40.

9 .

Rutten EPA, Lenaerts K, Buurman WA, Wouters EFM. Disturbed intestinal integrity in patients with COPD: effects of activities of daily living. Chest. 2014;145:245-52.

10.

Marsland BJ, Trompette A, Gollwitzer ES. The gut-lung axis in respiratory disease. Ann Am Thorac Soc. 2015;Suppl 2:150-6.

11.

Koh A, De Vadder F, Kovatcheva-Datchary P, Bäckhed F. From dietary fiber to host physiology: short-chain fatty acids as key bacterial metabolites. Cell. 2016;165:1332-45.

12.

Koeth RA, Wang Z, Levison BS, Buffa JA, Org E, Sheehy BT, Britt EB, Fu X, Wu Y, Li L, Smith JD, DiDonato JA, Chen J, Li H, Wu GD, Lewis JD, Warrier M, Brown JM, Krauss RM, Tang WH, Bushman FD, Lusis AJ, Hazen SL. Intestinal microbiota metabolism of L-carnitine, a nutrient in red meat, promotes atherosclerosis. Nat Med. 2013;19:576-85.

13.

Tang WH, Wang Z, Levison BS, Koeth RA, Britt EB, Fu X, Wu Y, Hazen SL. Intestinal microbial metabolism of phosphatidylcholine and cardiovascular risk. N Engl J Med. 2013;368:1575-84.

14.

Wang Z, Klipfell E, Bennett BJ, Koeth R, Levison BS, Dugar B, Feldstein AE, Britt EB, Fu X, Chung YM, et al. Gut flora metabolism of phosphatidylcholine promotes cardiovascular disease. Nature. 2011;472:57-63. 15.

Tang WH, Wang Z, Fan Y, Levison B, Hazen JE, Donahue LM, Wu Y, Hazen SL. Prognostic value of elevated levels of intestinal microbe-generated metabolite trimethylamine-N-oxide in patients with heart failure: Refining the gut hypothesis. J Am Coll Cardiol. 2014;64:1908-14.

16.

Trøseid M, Ueland T, Hov JR, Svardal A, Gregersen I, Dahl CP, Aakhus S, Gude E, Bjørndal B, Halvorsen B, Karlsen TH, Aukrust P, Gullestad L, Berge RK, Yndestad A. Microbiota- dependent metabolite trimethylamine-N-oxide is associated with disease severity and survival of patients with chronic heart failure. J Intern Med. 2015;277:717-26.

17.

Tang WH, Wang Z, Kennedy DJ, Wu Y, Buffa JA, Agatisa-Boyle B, Li XS, Levison BS, Hazen SL. Gut microbiota-dependent trimethylamine $\mathrm{N}$-oxide (TMAO) pathway contributes to both development of renal insufficiency and mortality risk in chronic kidney disease. Circ Res. 2015;116:448-55.

18.

Ottiger M, Nickler M, Steuer C, Odermatt J, Huber A, Christ-Crain M, Henzen C, Hoess C, Thomann R, Zimmerli W, Mueller B, Schuetz P. Trimethylamine-N-oxide (TMAO) predicts fatal outcomes in community acquired pneumonia patients without evident coronary artery disease. Eur J Intern Med. 2016;36:67-73. 19. 
Ottiger M, Nickler M, Steuer C, Bernasconi L, Huber A, Christ-Crain M, Henzen C, Hoess C, Thomann R, Zimmerli W, Mueller B, Schuetz P. Gut, microbiota-dependent trimethylamine-N-oxide is associated with long-term all-cause mortality in patients with exacerbated chronic obstructive pulmonary disease. Nutrition. 2018;45:135-41.

20.

Chen W, Thomas J, Sadatsafavi M, FitzGerald JM. Risk of cardiovascular comorbidity in patients with chronic obstructive pulmonary disease: A systematic review and meta-analysis. Lancet Respir Med. 2015;3:631-9.

21.

Patel AR, Hurst JR. Extrapulmonary comorbidities in chronic obstructive pulmonary disease: State of the art. Expert Rev Respir Med. 2011;5:647-62.

22.

Mammen MJ, Sethi S. COPD and the microbiome. Respirology. 2016;21:590-9. 23.

Anand S, Mande SS. Diet, Microbiota and Gut-Lung Connection. Front Microbiol. 2018;9:2147. 24.

Lee SH, Yun Y, Kim SJ, Lee EJ, Chang Y, Ryu S, Shin H, Kim HL, Kim HN, Lee JH. Association between cigarette smoking status and composition of gut microbiota: population-based cross-sectional study. J Clin Med. 2018;7:282.

25.

Benjamin JL, Hedin CR, Koutsoumpas A, Ng SC, McCarthy NE, Prescott NJ, Pessoa-Lopes P, Mathew CG, Sanderson J, Hart AL, Kamm MA, Knight SC, Forbes A, Stagg AJ, Lindsay JO, Whelan K. Smokers with active Crohn's disease have a clinically relevant dysbiosis of the gastrointestinal microbiota. Inflamm Bowel Dis. 2012;18:1092-100.

26.

Biedermann L, Zeitz J, Mwinyi J, Sutter-Minder E, Rehman A, Ott SJ, Steurer-Stey C, Frei A, Frei P, Scharl M, et al. Smoking cessation induces profound changes in the composition of the intestinal microbiota in humans. PLoS One. 2013;8:e59260.

27.

Allais L, Kerckhof FM, Verschuere S, Bracke KR, De Smet R, Laukens D, Van den Abbeele P, De Vos M, Boon N, Brusselle GG, Cuvelier CA, Van de Wiele T. Chronic cigarette smoke exposure induces microbial and inflammatory shifts and mucin changes in the murine gut. Environ Microbiol. 2016;18:1352-63. 28.

Bafadhel M, Pavord ID, Russell REK. Eosinophils in COPD: just another biomarker? Lancet Respir Med. 2017;5:747-59.

29.

Sze MA, Dimitriu PA, Suzuki M, McDonough JE, Campbell JD, Brothers JF, Erb-Downward JR, Huffnagle GB, Hayashi S, Elliott WM, Cooper J, Sin DD, Lenburg ME, Spira A, Mohn WW, Hogg JC. Host response to the lung microbiome in chronic obstructive pulmonary disease. Am J Respir Crit Care Med. 2015;192:438-45. 
30.

Wang Z, Bafadhel M, Haldar K, Spivak A, Mayhew D, Miller BE, Tal-Singer R, Johnston SL, Ramsheh MY, Barer MR, Brightling CE, Brown JR. Lung microbiome dynamics in COPD exacerbations. Eur Respir J. 2016;47:1082-92.

31.

Kolsum U, Donaldson GC, Singh R, Barker BL, Gupta V, George L, Webb AJ, Thurston S, Brookes AJ, McHugh TD, et al. Blood and sputum eosinophils in COPD; relationship with bacterial load. Respir Res. 2017;18:88.

32.

Ivanov II, Atarashi K, Manel N, Brodie EL, Shima T, Karaoz U, Wei D, Goldfarb KC, Santee CA, Lynch SV, Tanoue T, Imaoka A, Itoh K, Takeda K, Umesaki Y, Honda K, Littman DR. Induction of intestinal Th17 cells by segmented filamentous bacteria. Cell. 2009;139:485-98.

33.

Ivanov II, Frutos Rde L, Manel N, Yoshinaga K, Rifkin DB, Sartor RB, Finlay BB, Littman DR. Specific microbiota direct the differentiation of IL-17-producing T-helper cells in the mucosa of the small intestine. Cell Host Microbe. 2008;4:337-49.

34.

Bashir A, Miskeen AY, Hazari YM, Asrafuzzaman S, Fazili KM. Fusobacterium nucleatum, inflammation, and immunity: the fire within human gut. Tumour Biol. 2016;37:2805-10.

35.

Rasmussen M. Aerococcus an increasingly acknowledged human pathogen. Clin Microbiol Infect. 2016;22:22-7.

36.

lino C, Endo T, Mikami K, Hasegawa T, Kimura M, Sawada N, Nakaji S, Fukuda S. Significant decrease in Faecalibacterium among gut microbiota in nonalcoholic fatty liver disease: a large BMI- and sex-matched population study. Hepatol Int. 2019;13:748-56.

37.

Nguyen TT, Hathaway H, Kosciolek T, Knight R, Jeste DV. Gut microbiome in serious mental illnesses: A systematic review and critical evaluation. Schizophr Res. 2019 Sep 5. pii: S0920-9964(19)30382-2. doi: 10.1016/j.schres.2019.08.026. [Epub ahead of print].

38.

Ma B, Liang J, Dai M, Wang J, Luo J, Zhang Z, Jing J. Altered Gut Microbiota in Chinese Children With Autism Spectrum Disorders. Front Cell Infect Microbiol. 2019;9:40.

39.

Tito RY, Cypers H, Joossens M, Varkas G, Van Praet L, Glorieus E, Van den Bosch F, De Vos M, Raes J, Elewaut D. Brief Report: Dialister as a Microbial Marker of Disease Activity in Spondyloarthritis. Arthritis Rheumatol. 2017;69:114-21.

40 .

Global Initiative for Chronic Obstructive Lung Disease, Global strategy for diagnosis, management, and prevention of chronic obstructive pulmonary disease. GOLD. (2017). 


\section{Figures}

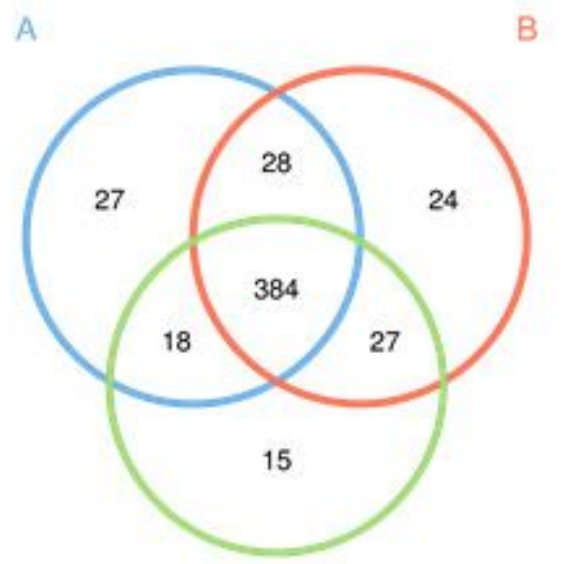

\section{Figure 1}

OTU Venn diagram. A: stage 1, B: stage 2, C: stage 3+4; The numbers represent the number of OTUs unique or common to each sample or group.
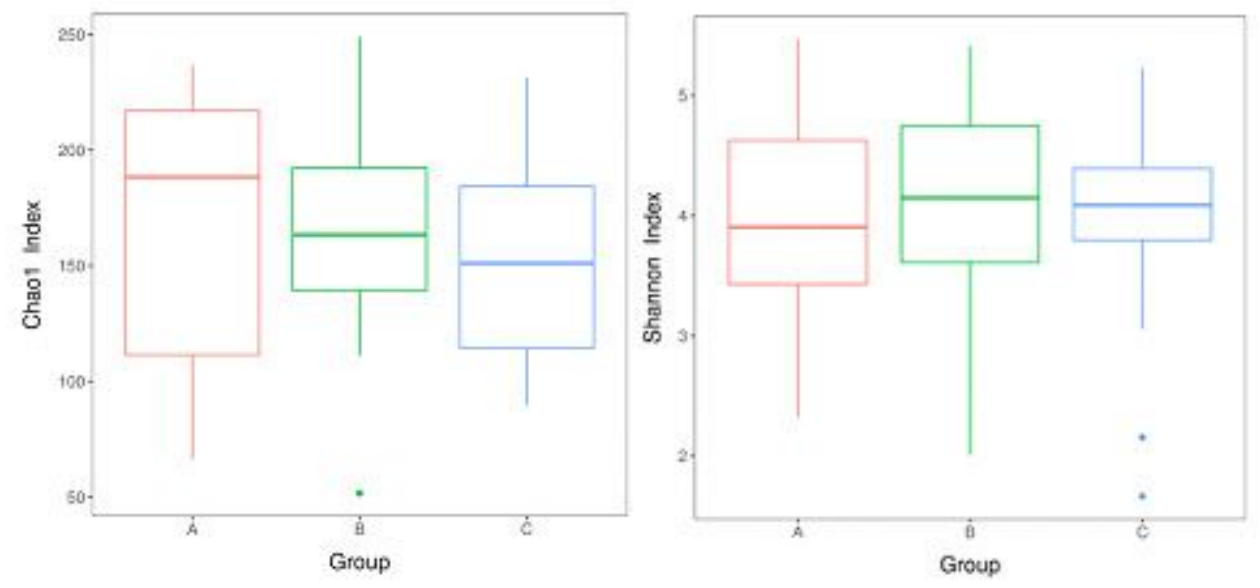

Figure 2

The indices for community richness of three COPD groups. Left: Chao1 index boxplot of each group. The $\mathrm{X}$-axis indicates the names of the groups and $\mathrm{Y}$-axis the Chao 1 index. Each box diagram shows the minimum, first quartile, medium, third quartile, and maximum values of the Chao 1 index of the corresponding sample. Right: Shannon index boxplot of each group. A: stage 1, B: stage 2, C: stage $3+4$. 


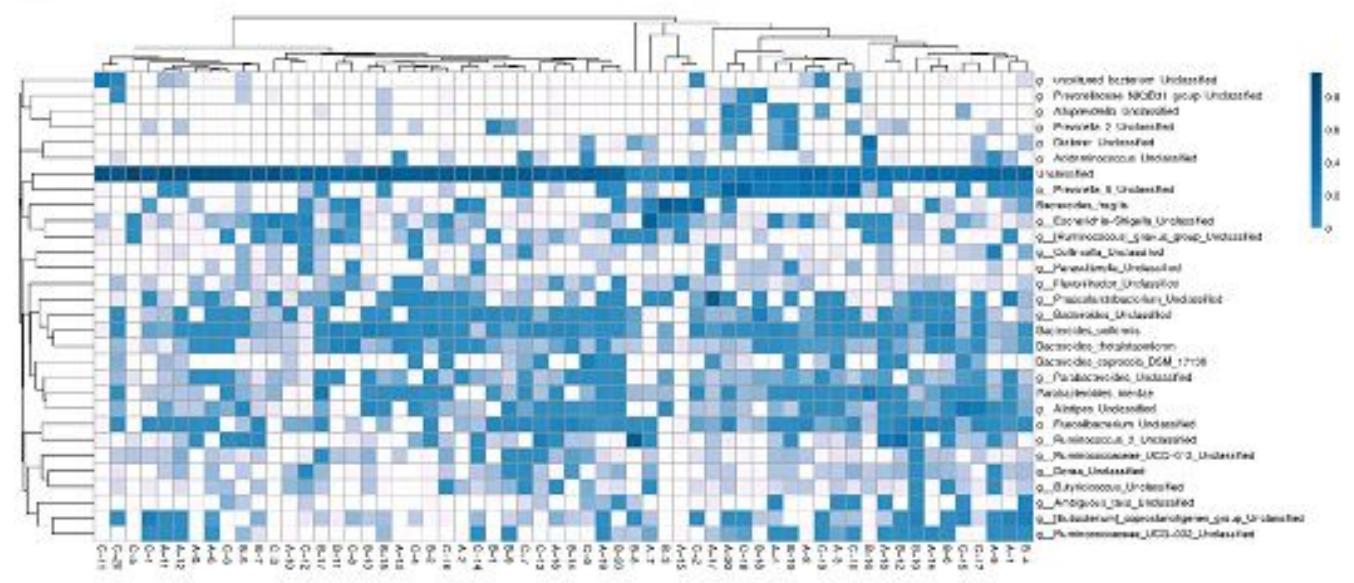

b

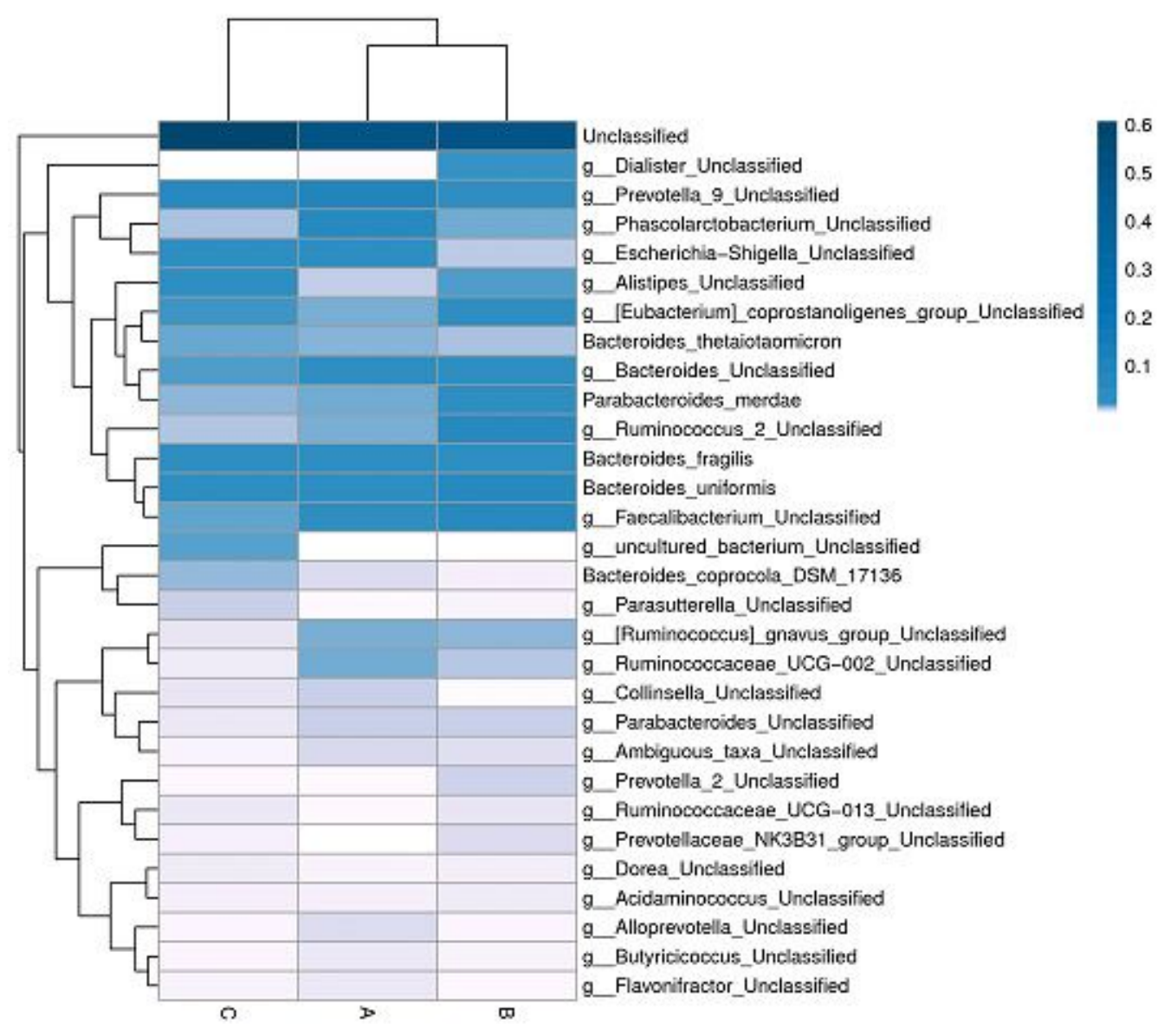

Figure 3

Species distribution heatmap. a: Cluster of samples. b: Cluster of groups. The columns represent samples and/or groups and the rows represent species. The dendrogram above the heatmap is the cluster result of the samples and/or groups and the dendrogram to the left is the species cluster. The colours in the heat map represent the relative abundance of the corresponding species in the corresponding sample or group. 


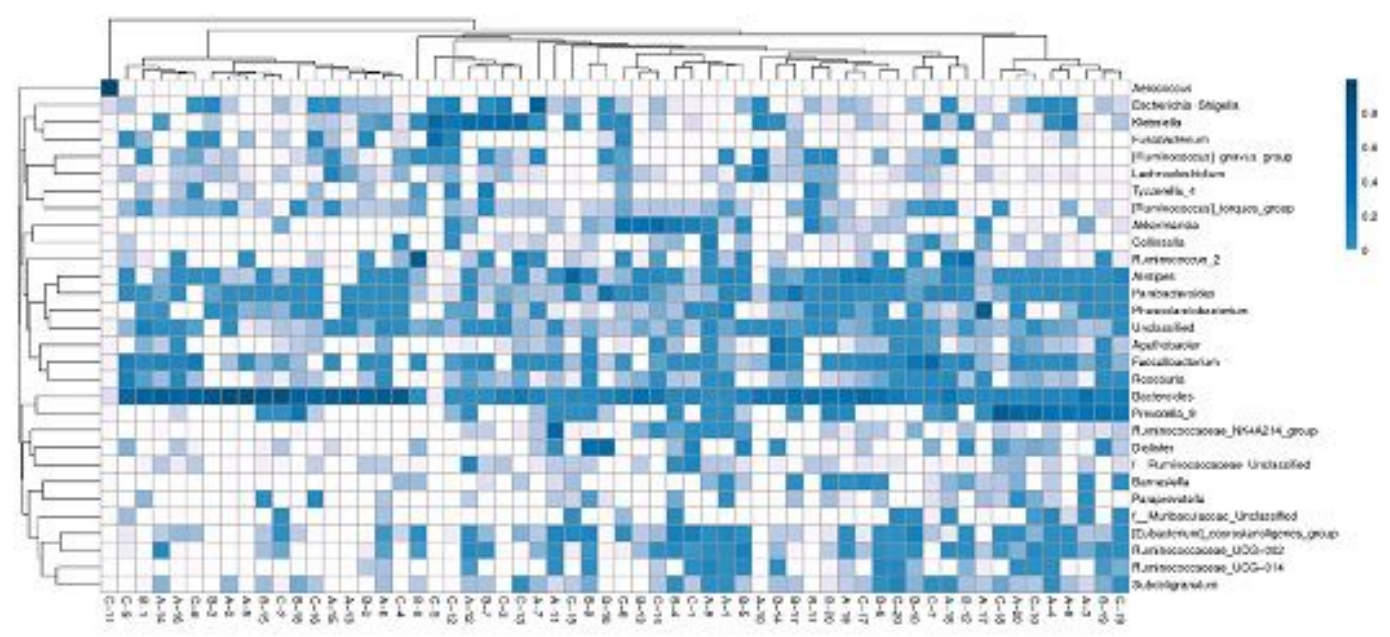

b

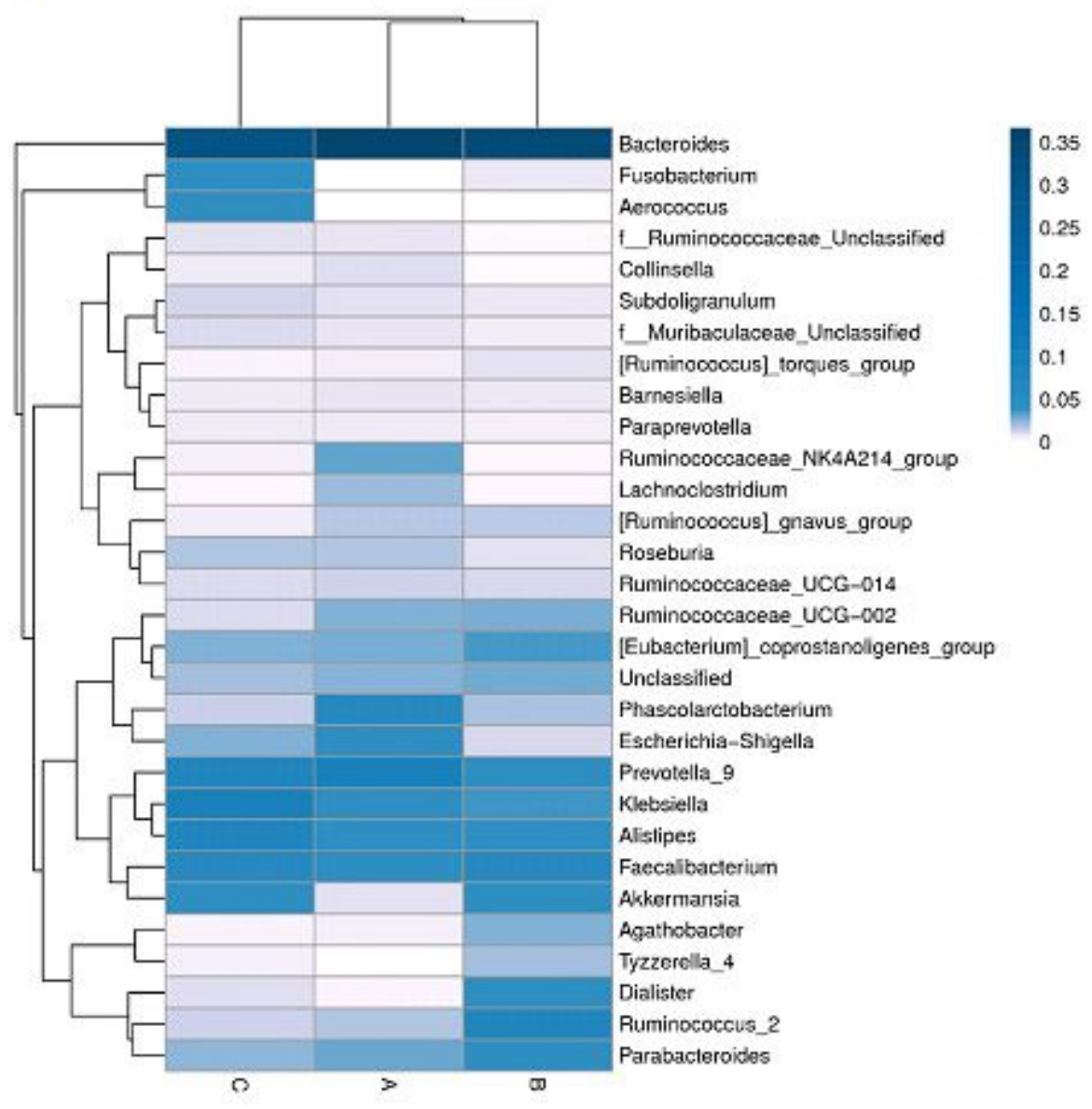

Figure 4

Genus distribution heatmap. a: Cluster of samples. b: Cluster of groups. The columns represent samples and/or groups and the rows represent genus. The dendrogram above the heatmap is the cluster result of the samples and/or groups and the dendrogram to the left is the genus cluster. The colours in the heat map represent the relative abundance of the corresponding genus in the corresponding sample or group. 

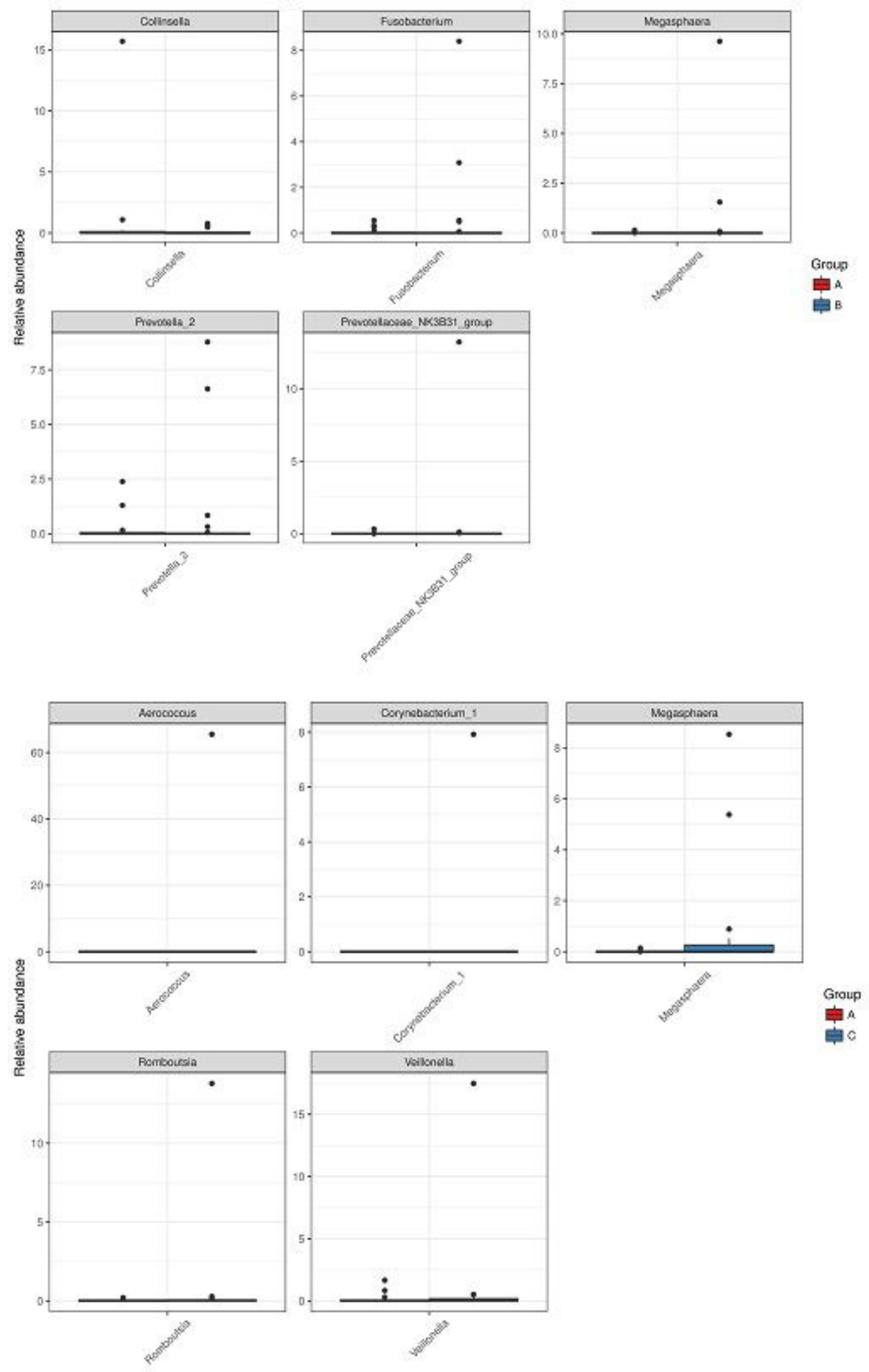

\section{Figure 5}

Abundance distributions of the five strains with the largest between-group differences. Top: group A vs. group B; middle: group A vs. group C; bottom: group B vs. group C. 


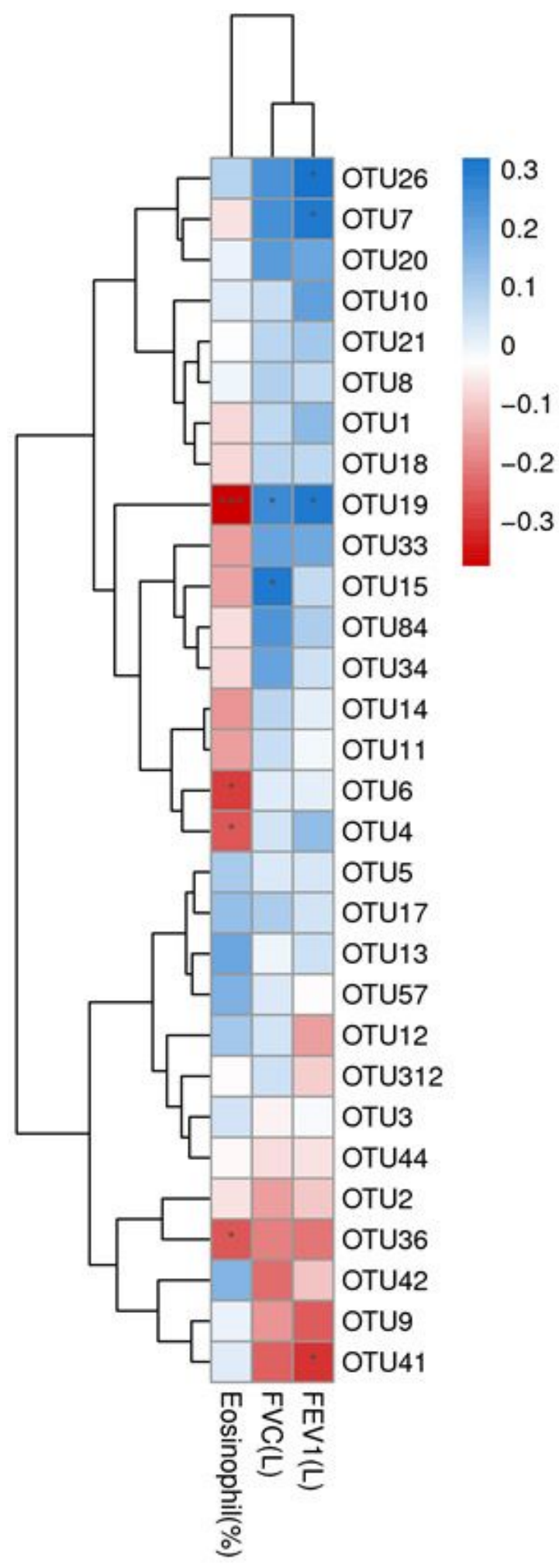

Figure 6

Heatmap of Spearman correlation between OTUs and eosinophil and pulmonary function. Spearman correlation coefficient $(r)$ ranges between -1 and $1 . r>0$ indicates positive correlation and $r<0$ negative correlation. ${ }^{\star} p=0.01-0.05,{ }^{* \star *} p<0.001$. OTU 26: g_Ruminococcaceae_UCG-002, s_uncultured organism; OTU 7: g_Faecalibacterium, s_Ambiguous taxa; OTU 19: Bacteroides sp.; OTU 15: g_Bacteroides, 
s_unidentified; OTU 6: Bacteroides sp.; OTU 4: Parabacteroides_merdae; OTU 36: Bacteroides sp.; OTU 41: Fusobacterium sp. IDs of other non-significant OTUs are listed in Additional file 1: Table S1.

\section{Supplementary Files}

This is a list of supplementary files associated with this preprint. Click to download.

- Figures1.docx

- TableS1otutaxatable.xls

- Tables2PvalueCol.xls 